The primary outcome measure was numbers of culture positive EOS cases where the calculator did not recommend empirical antibiotics. If the NICE guidelines would not have recommended treatment either this was not classified as a 'miss'. Risk of bias was assessed using QUADAS-2.

Data were pooled using a random effect meta-analysis, quantifying heterogeneity using $\mathrm{I}^{2}$. A subgroup analysis was performed using data from studies of babies exposed to chorioamnionitis.

Results Eleven studies were eligible. There were 75 EOS cases and a minimum of 14 , and a maximum of 22 cases where use of the calculator would have resulted in delayed or missed treatment, compared to if NICE guidelines were followed.

The probability of 'calculator' delayed or missed treatment for an EOS case (additional to cases missed by following NICE guidelines) were best case 0.19 [95\% confidence intervals $\left.0.11-0.29, \mathrm{I}^{2} 0 \%\right]$, worst case 0.31 [95\% CI 0.17 $\left.0.49, \mathrm{I}^{2} 37 \%\right]$.

The probability of missing cases was significantly $(p=0.03)$ more in babies exposed to chorioamnionitis, up to $0.56[95 \%$ CI: [0.25, 0.82], I² 0\%].

All included studies had a low/moderate probability of bias. Conclusion A substantial proportion of EOS cases were missed by the calculator. Further evaluation of the calculator is recommended before it could be safely introduced into UK clinical practice.

\section{G562(P) LIFESTART RESUSCITATION PLATFORM TO ENABLE DEFERRED CORD CLAMPING IN PRETERM INFANTS $<32$ WEEKS}

ES Hoyle, S Hirani, S Ogden, J Deeming, CW Yoxall. Neonatal Unit, Liverpool Women's Hospital, Liverpool, UK

\subsection{6/archdischild-2020-rcpch.480}

Aims Randomised controlled trials have demonstrated improved survival with deferred cord clamping (DCC) at preterm birth.

We adopted the use of Lifestart trolley (LS) in preterm deliveries at our centre in March 2013 to enable stabilisation of babies with an intact umbilical cord. Initial compliance with our guideline was poor.

A quality improvement project was undertaken with the aim of increasing LS use at delivery in preterm babies with 2 minutes DCC.

Methods A quality improvement programme from April 2018April 2019 was undertaken using Plan, See, Do, Act (PDSA) cycles. Data were reviewed from 113 consecutive preterm ( $<32$ weeks) deliveries to identify whether LS was used and whether 2 minutes DCC occurred in eligible infants. Episodes of non-compliance were analysed, causes established and interventions implemented to improve compliance rates. Data collected was distributed via alternate monthly newsletters to staff, including lessons learnt from the reviews of noncompliance.

Results The use of Lifestart was noted to increase progressively through the period to almost 80\% in April 2019.

Of those infants eligible, the rate of DCC also showed a progressive increase from $16 \%$ in the first three months to 80\% in the April 2019 (table 1).

This is in marked contrast to national data collected from Badgernet, which shows only $5 \%$ of preterm babies born

Abstract G562(P) Table 1
\begin{tabular}{lllllll}
\hline April-June & 18 & July-Sept & 18 & Oct-Dec 18 & Jan-March 19 & Apr 19 \\
\hline \% Lifestart used & 26.9 & 53.3 & 72.0 & 52.4 & 77.8 \\
\% Received DCC & 16 & 31.6 & 68.4 & 73.3 & 80 \\
\hline
\end{tabular}

before 32 weeks gestation have a documented period of DCC of 60 seconds or more.

Conclusions DCC at preterm birth increases survival. Despite this knowledge, there is limited implementation of this intervention across UK.

By undertaking regular PDSA cycles we have changed our practice of management at preterm deliveries.

The learning from the project enabled us to produce an instructional video (Available at http://bit.ly/LWHLifeStart) to support its use in our practise.

\section{G563(P) WHAT OUTCOMES IN NEONATAL RESEARCH ARE IMPORTANT TO HEALTHCARE WORKERS AND PARENTS IN NIGERIA?}

${ }^{1} \mathrm{~S}$ Read, ${ }^{2} \mathrm{~A}$ Jibril, ${ }^{3} \mathrm{~K}$ Tongo, ${ }^{3} \mathrm{~A}$ Abimbola, ${ }^{2}$ I Abdulkadir, ${ }^{4} \mathrm{H}$ Nabwera, ${ }^{5}$ I Sinha, ${ }^{5,6} \mathrm{~S}_{\text {Allen. }}$ ${ }^{1}$ International Public Health, Liverpool School of Tropical Medicine, Liverpool, UK; ${ }^{2}$ Faculty of Medicine, Ahmadu Bello University, Zaria, Nigeria; ${ }^{3}$ College of Medicine, University of Ibadan, Ibadan, Nigeria; ${ }^{4}$ Centre for Maternal and Newborn Health, Liverpool School of Tropical Medicine, Liverpool, UK; ${ }^{5}$ Alder Hey Children's NHS FT, Liverpool, UK; ${ }^{6}$ Clinical Sciences, Liverpool School of Tropical Medicine, Liverpool, UK

\subsection{6/archdischild-2020-rcpch.481}

Background There is a scarcity of information around the most important outcomes for research in neonatal units in low-resource settings. Identification of important outcomes, which reflect shared clinical decisions between healthcare workers and parents, would inform the development of a core outcome set (COS) for use in research.

Objective To identify the outcomes that are important to healthcare workers and parents of newborn babies in neonatal units in Nigeria.

Methods A Delphi process was conducted amongst healthcare workers from various centres in Nigeria to identify and rank outcomes they considered important. Semi-structured interviews were then undertaken with parents of babies (mostly mothers) admitted to two neonatal units to ascertain their opinions and rank the outcomes previously identified by clinicians that were particularly important to them.

Results Outcome domains of most importance identified by healthcare workers and parents were short-term morbidity, nutrition, mortality, bonding, quality of life, length of hospital stay, financial cost, and long-term outcomes (both medical and functional). Healthcare workers prioritised short-term morbidity, neonatal mortality, and long-term complications; however, there was substantial variation between physicians and nurses, the latter raising quality of life as important. Parents placed more emphasis on quality of life and functional status than health complications.

Conclusions Clinical trials in low-resource settings should consider the outcomes identified in our study. The opinions of parents and nurses (as opposed to just physicians) need to be considered in developing COS for neonatal research in lowresource settings. 